\title{
R2 Schistosoma mansoni antigens recognized by serological- proteomic approach as potentially able to distinguish the clinical status of endemic area residents
}

Fernanda Ludolf ${ }^{1}$, Isabela A Rodrigues ${ }^{1}$, Rodrigo Corrêa-Oliveira ${ }^{2}$, Andréa Gazzinelli ${ }^{3}$, Franco H. Falcone ${ }^{4}$, Rosiane A Silva-Pereira ${ }^{1}$, Guilherme C. Oliveira ${ }^{1}$

1 Centro de Pesquisas René Rachou, Fiocruz, MG, Genomics and Computational Biology Group, Belo Horizonte, Minas Gerais, Brazil

2 Centro de Pesquisas René Rachou, Fiocruz, MG, Cellular and Molecular Immunology Laboratory, Belo Horizonte, Minas Gerais, Brazil

3 Universidade Federal de Minas Gerais, Nursing School, Belo Horizonte, Minas Gerais, Brazil

4 The University of Nottingham, School of Pharmacy, Division of Molecular and Cellular Science, Nottingham, East Midlands, United Kingdom

Introduction: Despite intensive efforts towards schistosomiasis control, the disease is still one of the most prevalent in the world. Improvements in the diagnostic would represent a step forward to the transmission control, being a suitable diagnostic assay essential for epidemiological surveys. Diagnosis procedures require continuous adaptation to the disease control stage and assays that are simple, inexpensive, sensitive, specific and able to distinguish active from prior infection have yet to be developed. Progress on post-genomic technologies resulted in a more rational approaches for new biomarkers discovery.

Objective: To confirm Schistosoma mansoni antigens recognized by serological-proteomic approach as potentially able to distinguish the clinical status of the schistosomiasis endemic area residents.

Methodology: Immunoreactive proteins were identified by our group using $S$. mansoni adult worm protein extracts probed with pooled sera of infected (INF), non-infected individuals from endemic area (NE) and of non-infected individuals from non-endemic area (NI), in a twodimensional Western-blotting assay (2D-WB). For further validation of the rSmP40 as diagnostic candidate, WBs were conducted using the serum samples individually.

Results: A total of 47 different immunoreactive proteins were identified. Of these proteins, seven reacted exclusively to the INF sera pool, suggesting a possible use of this antigen panel for diagnostic purposes. Western-blotting (WB) with the $S$. mansoni recombinant protein Major Egg Antigen ( $\mathrm{rSmP} 40$ ), one of the INF sera pool exclusively recognized antigen, showed a similar serum recognition profile to the native protein in the 2D-WB. Of the 12 INF individually serum samples, $8(67 \%)$ 
recognized a protein band of approximately $40 \mathrm{KDa}$, corresponding to the rSmP40. None of the $8 \mathrm{NE}(0 \%)$ and neither of the $7 \mathrm{NI}(0 \%)$ serum samples were reactive to this same protein. The rSmP40 were reactive to the serum of individuals with different gender, age, TBM (total body minutes), and intensity of infection with low and high epg (eggs/gram of stool).

Conclusion: Once the proteins which make up the panel of exclusively INF sera pool recognized antigens were identified simultaneously in a same 2D-WB assay, it is proposed that all of them might have the same potential as the rSmP40 for the development of a new diagnostic test. These antigens may be used as a diagnostic kit based on the detection of at least one of them, being capable to distinguish the clinical status of the schistosomiasis endemic area residents.

Kaywords: Schistosoma Mansoni, Biomarkers, Immunodiagnostic, Proteomics 